\title{
28 Research Soure \\ Selinexor Improves the Anti-Cancer Effect of Tucidinostat on TP53 Wild-type Breast Cancer
}

Shengxi Xu ( $\nabla$ xushengxi_jx@yeah.net)

Jiujiang First People's Hospital https://orcid.org/0000-0002-9415-1170

\section{Yingfang Shi}

Jiujiang First People's Hospital

Sen Li

Jiujiang First People's Hospital

Research article

Keywords: HDAC, Tucidinostat, Selinexor, Breast cancer, p53

Posted Date: July 27th, 2021

DOI: https://doi.org/10.21203/rs.3.rs-729058/v1

License: (1) This work is licensed under a Creative Commons Attribution 4.0 International License.

Read Full License 


\section{Abstract}

Background: Histone deacetylase (HDAC) is closely related to the occurrence and development of breast cancer $(\mathrm{BC})$. Its inhibitor (HDACi) has been used to treat $\mathrm{BC}$, while the efficacy of clinical trials was not reached expectations. HDACi combined with other drugs may be an effective strategy. This study explored the effect of HDACitucidinostat combined with selinexor, anexportin 1 (XPO1) inhibitor, on BC cellsin vitro.

Methods: BC cell lines of MCF-7 (wt-TP53), MDA-MB-175 (wt-TP53), MDA-MB-134 (mut-TP53), T47D (mut-TP53) were cultured. The $\mathrm{IC}_{50}$ values of tucidinostat and selinexor on $\mathrm{BC}$ cells were calculated. The effects of tucidinostat and selinexor on proliferation, invasion and apoptosis of BC cells were observed accordingly. Western blotting was used to detect the protein expressions of p53, p21, Cyclin D1, Bcl-2 and Bax.

Results:Compared with mut-TP53 BC, both tucidinostat and selinexor showed better inhibitory activitiesonwt-TP53 BC including MCF-7 and MDA-MB-175. Tucidinostat combined with selinexor significantly improved the effects of tucidinostat alone on the proliferation and invasion inhibitions and apoptosis promotionsof MCF-7 and MDA-MB-175 cells in vitro. It also significantly enhanced the effects of tucidinostat on up-regulating the expression levels of acetyl-p53, nuclear p53, total p53, p21 and Bax, and down-regulating the expression levels of Cyclin D1 and Bcl-2 in MCF-7 or MDA-MB-175 cells.

Conclusion: Taken together, we believe that tucidinostat and selinexor are potentially effective drug combinations for the treatment of wt-TP53 BC, and the molecular mechanism may be throughenhancing the activity of p53 in the nucleus of $\mathrm{BC}$ cells to suppress proliferation and invasion and promote apoptosis of BC cells.

\section{Background}

According to reports, one in every 20 women in the world suffers from breast cancer (BC), and nearly $1 \%$ of $B C$ patients in male tumor cases $[1,2]$. BC is clinically classified into Luminal A, Luminal B, HER2 overexpression and triple-negative types according to the expression of estrogen receptor (ER), progesterone receptor (PR), human epidermal receptor 2 (HER2), which determines the corresponding treatment methods [3]. The pathogenesis of $\mathrm{BC}$ is not yet fully understood. Studies have shown that epigenetic modifications including DNA methylation, histone modifications, and non-coding RNA play important roles in the development of BC. Clinical diagnosis, prognostic evaluation and treatment methods based on epigenetic changes in $\mathrm{BC}$ have also been received extensive attentions and researches [4].

Histone acetylation modification is involved in regulating the expressions of tumor suppressor genes and oncogenes in BC, relating to apoptosis, metastasis, and growth of cancer cells [5]. Since acetylation is reversible, maintaining the balance of acetylation modification has become a strategy for the treatment of BC. Histone deacetylase (HDAC) is closely related to the occurrence and development of BC, and its 
inhibitors have been used to treat BC. However, monotherapy with HADC inhibitors (HDACi) did not show the expected therapeutic effects $[4,6]$. The combined use of cytotoxic chemotherapeutic agents or targeted drugs has been shown to improve the clinical outcomes of HDACi in the treatment of $\mathrm{BC}[7,8]$. In the preliminary experiments, we found that the exportin 1 (XPO1) inhibitor selinexor could obviously improve the effect of type I HDACi tucidinostat on proliferation inhibition of TP53 wild-type (wt-TP53) BC cells. In this study, we reported the therapeutic effects of selinexor combined with tucidinostat on BC in vitro and further explored the possible molecular regulation mechanism.

\section{Materials And Methods}

\section{Reagents}

Tucidinostat (dissolved in DMSO as $50 \mathrm{mg} / \mathrm{ml}$ ) and selinexor (dissolved in DMSO as $50 \mathrm{mg} / \mathrm{ml}$ ) were obtained commercially from Selleckchem (Houston, TX, USA). Annexin V-FITC apoptosis detection kit was obtained from eBioscience (San Diego, CA, USA). The antibodies of HDAC1, HDAC 2, HDAC3, XP01, acetyl-p53, p53, p21, Cyclin D1, B-cell lymphoma 2 (Bcl-2), Bcl-2-associated X (Bax) and GAPDH were purchased from abcam (Cambridge, UK).

\section{Cell Culture}

Human normal mammary epithelial cell line MCF 10A, human BC cell lines of MCF-7, MDA-MB-175, and T47D were all purchased from Procell (Wuhan, CHN). The human BC cell line of MDA-MB-134 was purchased from Fuheng Biology (Shanghai, CHN). The cells of MCF 10A and MCF-7 were grown in the specific medium provide by Procell at $37^{\circ} \mathrm{C}$ with $5 \% \mathrm{CO}_{2}(\mathrm{v} / \mathrm{v})$. The cells of MDA-MB- 175 were grown in Leibovitz's L-15 with $10 \%$ fetal bovine serum (FBS) and $1 \%$ penicillin-streptomycin at $37^{\circ} \mathrm{C}$ with air (v/v). The cells of MDA-MB-134 were grown in Leibovitz's L-15 with 20\% fetal bovine serum (FBS) and $1 \%$ penicillin-streptomycin at $37^{\circ} \mathrm{C}$ with air ( $\left.\mathrm{v} / \mathrm{v}\right)$. Medium was replaced two to three days and the cells were passaged when the cell adherence area reached $80 \%$ of the culture dish.

\section{Mtt Assay}

The $B C$ cells were treated with different concentrations of tucidinostat $(0,2.5,5,10,20$ and $40 \mu \mathrm{M})$ and/or Selinexor $(0,12.5,2.5,5,10$ and $20 \mu \mathrm{M})$ in medium. The cells viability was detected by $3-(4,5-$ dimethylthiazol-2-yl)-5-(3-carboxymethoxyphenyl)-2-(4-sulfophenyl)-2H-tetrazolium (MTT; Promega, WI, USA). Briefly, after $72 \mathrm{~h}$ treatment in 96-well plates, the cells were incubated with $20 \mu \mathrm{lMTT}(5 \mathrm{mg} / \mathrm{ml})$ in $100 \mu \mathrm{l}$ cell culture medium for $4 \mathrm{~h}$ at $37^{\circ} \mathrm{C}$, then the absorbance of each well was measured at a wavelength of $490 \mathrm{~nm}$.

\section{Cell Count}


The cells of MCF-7 and MDA-MB-175 were transfected with luciferase of mCherry to enable them to be read and photographed by Celigo (Nexcelom, Lawrence, MA, USA). The software of Celigo was used to count the number of cells, and a cell growth curve was drawn after 5 days of continuous observation.

\section{Cell Invasion Assay}

Briefly, $1 \times 10^{5}$ cells were plated in the upper chamber of transwell chamber (Millipore Corporation,Billerica[MA匹USA) which was coated with Matrigel (BD Biosciences, Franklin Lakes, NJ, USA) cultured with medium without FBS, while $0.5 \mathrm{ml}$ DMEM containing $10 \%$ FBS was added to the lower chamber. After culturing at $37^{\circ} \mathrm{C}$ for $24 \mathrm{~h}$, the cells in lower chamber were washed with PBS and fixed with $4 \%$ paraformaldehyde for $20 \mathrm{~min}$. Then cells were stained with $0.25 \%$ crystal violet (Macklin Inc.

Shanghai, China), which was dissolved in $20 \%$ methanol, for 20-45 min, and washed again with PBS for twice. Light microscope was used to observe and counted for 10 random fields per well. Cell counts are expressed as the mean number of cells per field of view.

\section{Apoptosis Assay}

Single-cell suspension was obtained after trypsin-EDTA incubating $10 \mathrm{~min}$. The cells were washed with chilled D-Hanks ( $\mathrm{pH}=7.2 \sim 7.4$ ), and incubated in Annexin-V binding buffer for $15 \mathrm{~min}$ at room temperature, which containing Annexin-V- FITC. Flow cytometry (Becton Dickinsonm, USA) was used to quantify the fluorescence of Annexin-V-FITC with a minimum of 10,000 cells counted for each group.

\section{Western Blotting}

Protein extraction reagents (Solarbio, Beijing, $\mathrm{CHN}$ ) were used to extract total protein and nuclear protein respectively according to the instructions provided by the manufacturer, then electrophoresed on $10 \%$ SDS-PAGE gel and transferred to PVDF membranes. $10 \%$ non-fat milk was used to block the PVDF membranes for $60 \mathrm{~min}$ and then incubated with primary detection antibodies at $4{ }^{\circ} \mathrm{C}$ for a night. After washing by TBST, the membranes were incubated with HRP-conjugated secondary antibodies, detected by enhanced chemiluminescence (ECL, Thermo Fisher Scientific, Waltham, MA, USA) and quantified with the Image $J$ v2.1.4.7 software (National Institutes of Health, Bethesda, MD, USA).

\section{Statistical analysis}

Data was presented as means \pm S.E.M and analysed by SPSS version 20.0 (IBM Corp., Armonk, NY, USA) for variance homogeneity test and one-way analysis of variance. $P<0.05$ was considered to indicate a statistically significant difference. Calcusyn software (Biosoft, Ferguson, MO and Cambridge, UK) was used to calculate the combination index $(\mathrm{Cl})$ of drug combination according to Chou-Talalay method [9], which quantitatively established additivity $(\mathrm{Cl}=0.9-1.1)$, synergy $(\mathrm{Cl}<0.9)$ and antagonism $(\mathrm{Cl}>1.1)[10]$, 
and the resulting values were utilized in the construction of a plot of $\mathrm{Cl}$ values over a range of affected fractions (Fa-Cl plot).

\section{Results}

\section{HDAC1, 2, 3 and XPO1 highly express in BC cells}

Western blotting was used to detect the protein expressions of HDAC1, 2, 3, the target protein of tucidinostat, and XPO1, the target protein of selinexor, in human normal breast cells MCF $10 \mathrm{~A}$ and BC cells MCF-7, MDA-MB-175, MDA-MB-134, T47D. As shown in Fig. 1, compared with MCF 10A, the protein expressions of HDAC1, 2, 3 and XP01 in MCF-7, MDA-MB-175, MDA-MB-134, and T47D cells were significantly up-regulated $(p<0.05)$.

\section{Effects of tucidinostat and selinexor on the BC cells viability}

The MTT assay was used to detect the cells viability of MCF-7, MDA-MB-175, MDA-MB-134, and T47D at different concentrations of tucidinostat and selinexor, and the corresponding half maximal inhibitory concentration $\left(\mathrm{IC}_{50}\right)$ values were calculated and showed in Fig. 2. The $\mathrm{IC}_{50}$ values of tucidinostat on MCF7, MDA-MB-175, MDA-MB-134, and T47D were 13.6, 9.2, 24.4 and $19.3 \mu \mathrm{M}$, respectively. The $\mathrm{IC}_{50}$ values of selinexor on MCF- 7, MDA-MB-175, MDA-MB-134 and T47D were 2.6, 6.8, 9.7 and 12.0 $\mu$ M, respectively. It can be seen that the $\mathrm{IC}_{50}$ values of tucidinosta or selinexor on MCF-7 and MDA-MB-175 cells were lower than those on MDA-MB-134 and T47D cells, suggesting that the cytotoxic effects of tucidinosta and selinexor on MCF-7 and MDA-MB-175 cells were better than those on MDA-MB-134 and T47D cells. In addition, selinexor obviously had lower $\mathrm{IC}_{50}$ values for all types of $\mathrm{BC}$ cells compared with tucidinostat.

The MTT assay was also used to observe the effect of combined intervention of tucidinostat and selinexor on the cells viability of MCF-7, MDA-MB-175, MDA-MB-134, and T47D. As shown in Fig. 3, for MCF-7 and MDA-MB-175 cells, the inhibitory effects of tucidinostat combined with selinexor on cells viability were significantly better than that of tucidinostat or selinexor alone $(p<0.05)$. Their corresponding $\mathrm{Cl}$ plot analysis showed synergistically inhibition at the majority of concentrations. However, for MDA-MB-134 and T47D cells, the inhibitory effects of tucidinostat combined with selinexor on cells viability did not show better than that of single-agent intervention significantly $(p>0.05)$. Their corresponding $\mathrm{Cl}$ plot analysis also showed additively inhibition at the majority of concentrations. The combined drug treatment of $20 \mu \mathrm{M}$ tucidinostat and $10 \mu \mathrm{M}$ Selinexor, which combination has lowest $\mathrm{Cl}$ values, marked a transition from drug concentrations that prevented the growth of MCF-7 and MDA-MB175 only to a concentration that effectively prevented the growth of cancer cells. Therefore, we studied the combined administration of this corresponding concentration later.

\section{Effects of tucidinostat combined with selinexor on the proliferations of wt-TP53 BC cells}

To observe the effects of continuous intervention of tucidinostat combined with selinexor for 5 days on the proliferations of wt-TP53 BC cells. As shown in Fig. 4, tucidinostat or selinexor alone could 
significantly inhibit the number of MCF-7 and MDA-MB-175 cells after 5 days of intervention $(p<0.05$ vs. Control). Among them, the inhibitory effects of selinexor on the proliferations of MCF-7 and MDA-MB-175 cells was significantly better than that of tucidinostat ( $p<0.05 \mathrm{vs}$. Tucidinostat). The intervention of tucidinostat combined with selinexor could significantly enhance the proliferation inhibitory effects on MCF-7 and MDA-MB-175 cells ( $p<0.05$ vs. Selinexor).

\section{Effects of tucidinostat combined with selinexor on the invasion of wt-TP53 BC cells}

Transwell was used to observe the effects of tucidinostat combined with selinexor on the invasion of MCF-7 and MDA-MB-175 cells after $24 \mathrm{~h}$ intervention. As shown in Fig. 5, tucidinostat or selinexor alone could significantly inhibit the invasion of MCF-7 and MDA-MB-175 cells after $24 \mathrm{~h}$ interventions $(p<0.05$ vs. Control). Among them, the inhibitory effects of selinexor on the invasion of MCF-7 and MDA-MB-175 cells were significantly better than that of tucidinostat ( $p<0.05 \mathrm{vs}$. Tucidinostat). The combination of selinexor could significantly enhance the cell invasion inhibitory effects of tucidinostat on MCF-7 and MDA-MB-175 cells $(p<0.05)$.

\section{Effects of tucidinostat combined with selinexor on the apoptosis of wt-TP53 BC cells}

Flow cytometry was used to observe the effects of tucidinostat combined with selinexor on the apoptosis of MCF-7 and MDA-MB-175 cells after 5 days of intervention. As shown in Fig. 6 , tucidinostat or selinexor alone could significantly promote the apoptosis of MCF-7 and MDA-MB-175 cells ( $p<0.05$ vs. Control). Among them, the promoting effects of selinexor on the apoptosis of MCF-7 and MDA-MB-175 cells were significantly better than that of tucidinostat ( $p<0.05 \mathrm{vs}$. Tucidinostat). The combination of selinexor could significantly enhance the apoptosis promotion effects of tucidinostat on MCF-7 and MDA-MB-175 cells $(p<0.05)$.

\section{Effects of tucidinostat combined with selinexor on the protein expressions in wt-TP53 BC cells}

Western blotting was used to detect the expression levels of acetylated p53, nuclear p53, total p53, p21, Cyclin D1, Bcl-2 and Bax in MCF-7 and MDA-MB-175 cells after 5 days of intervention by tucidinostat and selinexor. As shown in Fig. 7, tucidinostat intervention could significantly up-regulate the expression levels of acetylated p53, nuclear p53 and total p53 in MCF-7 and MDA-MB-175 cells ( $p<0.05$ vs. Control), and significantly promote the expressions of p21 and Bax, while the expressions of Cyclin D1 and Bcl-2 protein were suppressed ( $p<0.05 \mathrm{vs}$. Control). Selinexor had no significant effect on the expression of acetylated p53 ( $p>0.05$ vs. Control), but its effect on up-regulating the expression of nuclear P53 protein was stronger than that of tucidinostat ( $p<0.05$ vs. Tucidinostat). Selinexor could also significantly upregulate the expressions of $\mathrm{P} 21$ and Bax, and down-regulate the expressions of Cyclin D1 and Bcl-2 ( $p<$ 0.05 vs. Control). The combination of tucidinostat and selinexor could further affect the expressions of above protein accordingly ( $p<0.05$ vs. the groups of Tucidinostat or Selinexor).

\section{Discussion}


Tucidinostat is a selective inhibitor of benzamide HDAC subtypes, which mainly targets subtypes 1, 2, 3 of class I HDACs and subtype 10 of class Ilb HDACs, and has a regulatory effect on abnormal epigenetic functions of tumors [11]. Tucidinostat induces chromatin remodeling by inhibiting HDAC to increase the acetylation level of chromatin histones, which results in changes in genes expressions of multiple signaling pathways, thereby inhibiting tumor cell cycle and inducing apoptosis [12]. It can also induce and enhance the tumor killing effect mediated by natural killer cells (NK) and antigen-specific cytotoxic T cells $(C T L)[13,14]$. Tucidinostat is approved for the treatment of peripheral T-cell lymphoma in China. In terms of the treatment of $\mathrm{BC}$, the effect of tucidinostat monotherapy is not satisfactory, but its combination with aromatase inhibitors has been successful in the large-scale phase 3 clinical trial and has been approved by National Medical Products Administration of China for patients with estrogen receptor-positive clinically advanced or metastatic $B C$ [8]. This may be related to the down-regulation of non-estrogen-dependent growth factor signaling pathways by tucidinostat and the restoration of sensitivity to anti-estrogen drugs [15]. In this study, four ER+Her2- ${ }^{-}$BC cell lines, MCF-7, MDA-MB-175, MDA-MB-134, and T47D, were selected as the research objects, which were divided into wt-TP53 (MCF-7 and MDA-MB-175) and mut-TP53 (MDA-MB-134 and T47) groups. We observed that the four BC cell lines all have higher levels of HDAC1, 2, and 3 expressions compared with normal breast cells. Tucidinostat showed more significant inhibitory effects on proliferations of wt-TP53 BC cells than mut-TP53 BC cells. We speculated that wt-TP5 may be the applicable type of BC for tucidinostat.

The nucleus is the regulatory center of cell genetics and metabolism. A large number of nuclear pores are distributed in the nuclear membrane of the cell nucleus. In addition to transporting mRNA, rRNA and other genetic material to complete basic functions such as translation, nuclear pores also transport many regulatory proteins, such as p53, forkhead box, and the likes. Among them, any molecule larger than 40 $\mathrm{kDa}$ needs the help of a special transporter to move between the nucleus and the cytoplasm [16]. Protein nuclear export is mainly regulated by XP01. With the help of Ran-GTP, XPO1 binds to cargo proteins by recognizing nuclear export signals. Hundreds of cellular proteins and many viral accessory proteins are known to carry nuclear export signals that can be recognized by XP01 [17]. A series of DNA mutations are produced during cell division. Some tumor suppressor proteins in the nucleus, such as p53, monitor DNA mutations and initiate protective mechanisms, prompting cancer cells to enter the process of apoptosis. Studies have shown that XPO1 is the only nuclear export transporter involved in the transport of tumor suppressors and growth regulators [18]. In cancer cells, XPO1 is generally overactive, leading to abnormal output of many important tumor suppressor factors to the cytoplasm, which depriving them of normal function [19]. Studies suggested that excessive nuclear export may be one of the key factors leading to tumorigenesis and chemotherapy resistance [20]. Drugs in the family of selective nuclear export inhibitors, including selinexor and related drugs verdinexor (KPT-335), can effectively block XPO1mediated nuclear export, thereby preserving the nuclear localization of tumor suppressors [21]. Selinexor has been evaluated in basic research and clinical trials of multiple cancer types [22], and has been approved by the U.S. Food and Drug Administration for refractory multiple myeloma [23]. The research of selinexor in the treatment of $\mathrm{BC}$ has been reported. Arango et al. observed the inhibitory effects of selinexor on the proliferations of $26 \mathrm{BC}$ cell lines with different subtypes, and confirmed that selinexor is a 
promising drug for the treatment of triple-negative BC [24]. However, a phase II clinical trial reported that selinexor was well tolerated in patients with advanced triple-negative $B C$, but did not produce an objective response [25]. In this study, we observed that MCF-7, MDA-MB-175, MDA-MB-134, and T47D all have higher levels of XPO1 expressions compared with normal breast cells. Selinexor alone had a poor inhibitory effect on the proliferation of the four types of BC cells, which was basically consistent with the results of Arango et al. [24]. However, it is interesting that the proliferation inhibitory effects on wt-TP53 $B C$ cells were significantly enhanced by combining with tucidinostat. The above results suggest that nuclear export signaling pathway and histone acetylation modification may have cross-talk in wt-TP53 $\mathrm{BC}$, and targeting of the two signaling pathways together may be a potential effective strategy for the treatment of wt-TP53 BC.

TP53 is considered to be a tumor suppressor gene, the p53 protein synthesized after transcription and translation of it participates in the coordination of cell cycle arrest, apoptosis, aging, metabolism, differentiation, angiogenesis and other cellular responses, and plays important roles in regulating cell integrity and homeostasis [26]. However, TP53 gene mutation is a common genetic event in most human tumors, and more than $30 \%$ of BC patients have TP53 mutations [27]. Acetylation helps p53 to sense and integrate various internal and external cellular stress signals, such as changes in oncogene activation, and separate and translocate from the E3 ubiquitin ligase MDM2 to the central transcription factor in the nucleus to regulate multiple downstream target genes, and then regulate the cell cycle progression and cell death [28]. It has been reported that 13 lysine residues located at the C-terminal of p53 are the main acetylation modification sites, and lysine 120 (K120) located in the DNA binding domain has also been confirmed to play a key role in promoting p53-mediated apoptosis $[29,30]$. The acetylation modification of p53 is completed by the CBP/p300 of HATs or the TIP60/ hMOF of MYST family, and the deacetylation modification is controlled by HDAC [31]. Studies have confirmed that HDAC1, HDAC2, and HDAC3 are involved in the deacetylation process of p53 [32-34]. The destruction of deacetylation at different sites of p53 by inhibiting HDAC may affect the binding activity of sequence-specific DNA, thereby activating target genes or altering nuclear export, coactivator recruitment or p53 stability. Studies have shown that the use of HDAC inhibitors over-acetylates the key residues of p53, enhance the stability of p53, promote cell cycle arrest and pro-apoptotic gene expression [35]. This study showed that tucidinostat could significantly promote apoptosis of MCF-7 and MDA-MB-175 cells. It also up-regulated the expression levels of acetylation p53, nuclear p53, total p53, p21 and Bax, and down-regulated the expressions of Cyclin D1 and Bcl-2. These results suggest that tucidinostat can promote p53 acetylation in wt-TP53 BC cells to stabilize the activity of p53 protein, thereby regulating downstream apoptosis - related proteins and promoting $\mathrm{BC}$ apoptosis.

In cancer cells, nuclear export protein is abnormally active, resulting in excessive export of tumor suppressor protein to the cell nucleus, which cannot exert its anti-tumor effect. Among the nuclear export proteins, only XP01 is responsible for the nuclear export of p53 [36]. Overactive XP01 can cause p53 to translocate into the cytoplasm, causing it to lose its function and promote the development of cancer [37]. XP01-mediated abnormal output of p53 has been found in a variety of cancers, and is associated with poor prognosis or drug resistance in patients [37]. Based on this, XPO1 has also become an effective 
target for tumor treatment. In this study, we found that the XPO1 inhibitor selinexor could enhance the effects of tucidinostat on inhibiting the proliferation and promoting apoptosis of wt-TP53 BC cells. Further western blotting results showed that selinexor intervention could significantly up-regulate the protein level of p53 in the nucleus. We speculated that in wt-TP53 BC cells, tucidinostat inhibits the deacetylation of p53 by targeting HDACs, improves the stability and activity of p53 protein, while selinexor can reduce the nuclear export of p53 protein by targeting XPO1, and further increase the amount of p53 protein in the nucleus, which enhancing the anti-BC effect of tucidinostat.

\section{Conclusion}

In this study, we found that compared with the mut-TP53, tucidinostat exhibited better proliferation inhibitory effects on wt-TP53 BC cells such as cell lines of MCF-7 and MDA-MB-175. Tucidinostat could significantly inhibit invasion and promote apoptosis of MCF-7 and MDA-MB-175 cells, up-regulated the expression levels of acetylation p53, nuclear p53, total p53, p21 and Bax, and down-regulated the expressions of Cyclin D1 and Bcl-2, suggesting that the anti-BC effect of tucidinostat may be mediated through the p53 signaling pathway. The combination of XPO1 inhibitor selinexor and tucidinostat enhanced above effects of tucidinostat on wt-TP53 BC cells. We believe that the combination of tucidinostat and selinexor is a potentially effective drug combination for the treatment of wt-TP53 BC, and the molecular mechanism may be through increasing the p53 activity in the nucleus of BC cells to inhibit cancer cell proliferation and induce apoptosis.

\section{Declarations}

\section{Ethics approval and consent to participate}

Not applicable.

\section{Consent for publication}

Not applicable.

\section{Availability of data and materials}

All data generated or analyzed during this study are included in thispublished article, or available upon reasonable request from thecorresponding author.

\section{Competing interests}

The authors declare no competing interests.

\section{Funding}


This research did not receive any specific grant from funding agencies in the public, commercial, or notfor-profit sectors.

\section{Authors' contributions}

YS conceived and designedthe study. YSand SX developed the methodology and acquiredthe data. SL analyzed and interpreted the data forpresentation. YS wrote the manuscript. All authors read and

\section{Acknowledgements}

Not applicable.

\section{References}

1. Britt KL, Cuzick J, Phillips K-A. Key steps for effective breast cancer prevention. Nat Rev Cancer. 2020;20:417-36.

2. Methamem M, Ghadhab I, Hidar S, Briki R. Breast cancer in men: a serie of 45 cases and literature review. Pan Afr Med J. 2020;36:183.

3. Barzaman K, Karami J, Zarei Z, Hosseinzadeh A, Kazemi MH, Moradi-Kalbolandi S, et al. Breast cancer: Biology, biomarkers, and treatments. Int Immunopharmacol. 2020;84:106535.

4. Sher G, Salman NA, Khan AQ, Prabhu KS, Raza A, Kulinski M, et al. Epigenetic and breast cancer therapy: Promising diagnostic and therapeutic applications. Semin Cancer Biol. 2020;S1044579X:30181-4.

5. Guo P, Chen W, Li H, Li M, Li L. The Histone Acetylation Modifications of Breast Cancer and their Therapeutic Implications. Pathol Oncol Res. 2018;24:807-13.

6. Ho TCS, Chan AHY, Ganesan A. Thirty Years of HDAC Inhibitors: 2020 Insight and Hindsight. J Med Chem. 2020;63:12460-84.

7. Yardley DA, Ismail-Khan RR, Melichar B, Lichinitser M, Munster PN, Klein PM, et al. Randomized Phase II, Double-Blind, Placebo-Controlled Study of Exemestane with or without Entinostat in Postmenopausal Women with Locally Recurrent or Metastatic Estrogen Receptor-Positive Breast Cancer Progressing on Treatment with a Nonsteroidal Aromatase Inhibitor. J Clin Oncol. 2013;31:2128-35.

8. Jiang Z, Li W, Hu X, Zhang Q, Sun T, Cui S, et al. Tucidinostat plus exemestane for postmenopausal patients with advanced, hormone receptor-positive breast cancer (ACE): a randomised, double-blind, placebo-controlled, phase 3 trial. Lancet Oncol. 2019;20:806-15.

9. Chou TC, Talalay P. Quantitative analysis of dose-effect relationships: the combined effects of multiple drugs or enzyme inhibitors. Adv Enzyme Regul. 1984;22:27-55.

10. Jones GS, Yu F, Zeynalzadegan A, Hesselgesser J, Chen X, Chen J, et al. Preclinical evaluation of GS9160, a novel inhibitor of human immunodeficiency virus type 1 integrase. Antimicrob Agents Chemother. 2009;53:1194-203. 
11. Shi Y, Jia B, Xu W, Li W, Liu T, Liu P, et al. Chidamide in relapsed or refractory peripheral T cell lymphoma: a multicenter real-world study in China. J Hematol Oncol. 2017;10:69.

12. Gao S, Li X, Zang J, Xu W, Zhang Y. Preclinical and Clinical Studies of Chidamide (CS055/HBI-8000), An Orally Available Subtype-selective HDAC Inhibitor for Cancer Therapy. Anticancer Agents Med Chem. 2017;17:802-12.

13. Yao Y, Zhou J, Wang L, Gao X, Ning Q, Jiang M, et al. Increased PRAME-specific CTL killing of acute myeloid leukemia cells by either a novel histone deacetylase inhibitor chidamide alone or combined treatment with decitabine. PLoS One. 2013;8:e70522.

14. Shen L, Pili R. Class I histone deacetylase inhibition is a novel mechanism to target regulatory $T$ cells in immunotherapy. Oncoimmunology. 2012;1:948-50.

15. Y Z, YN W, K Z, J Z, Z N. Chidamide reverses epidermal growth factor induced endocrine resistance in estrogen receptor-positive breast cancer. J Shenzhen Univ Sci Eng. 2018;35:339-44.

16. Muqbil I, Azmi AS, Mohammad RM. Nuclear export inhibition for pancreatic cancer therapy. Cancers (Basel). 2018;10:138.

17. Mathew C, Ghildyal R. CRM1 Inhibitors for Antiviral Therapy. Front Microbiol. 2017;8:1171.

18. Parikh K, Cang S, Sekhri A, Liu D. Selective inhibitors of nuclear export (sine)--A novel class of anticancer agents. J Hematol Oncol. 2014;7:78.

19. Uddin MH, Zonder JA, Azmi AS. Exportin 1 inhibition as antiviral therapy. Drug Discov Today. 2020;25:1775-81.

20. Tan DSP, Bedard PL, Kuruvilla J, Siu LL, Razak ARA. Promising SINEs for embargoing nuclearcytoplasmic export as an anticancer strategy. Cancer Discov. 2014;4:527-37.

21. Conforti F, Wang Y, Rodriguez JA, Alberobello AT, Zhang Y-W, Giaccone G. Molecular pathways: anticancer activity by inhibition of nucleocytoplasmic shuttling. Clin Cancer Res. 2015;21:4508-13.

22. Wang AY, Liu H. The past, present, and future of CRM1/XPO1 inhibitors. Stem Cell Investig. 2019;6:6.

23. Chari A, Vogl DT, Gavriatopoulou M, Nooka A. Oral Selinexor-Dexamethasone for Triple-Class Refractory Multiple Myeloma. N Engl J Med. 2019;381:727-38.

24. Arango NP, Yuca E, Zhao M, Evans KW, Scott S, Kim C, et al. Selinexor (KPT-330) demonstrates antitumor efficacy in preclinical models of triple-negative breast cancer. Breast Cancer Res. 2017;19:93.

25. Shafique M, Ismail-Khan R, Extermann M, Sullivan D, Goodridge D, Boulware D, et al. A Phase II Trial of Selinexor (KPT-330) for Metastatic Triple-Negative Breast Cancer. Oncologist. 2019;24:887-e416.

26. Vousden KH, Lane DP. p53 in health and disease. Nat Rev Mol Cell Biol. 2007;8:275-83.

27. Lacroix M, Toillon R-A, Leclercq G. p53 and breast cancer, an update. Endocr Relat Cancer. 2006;13:293-325.

28. Duffy MJ, Synnott NC, O'Grady S, Crown J. Targeting p53 for the treatment of cancer. Semin Cancer Biol. 2020;S1044-579X:30160-7.

29. Brooks CL, Gu W. The impact of acetylation and deacetylation on the p53 pathway. Protein Cell. 2011;2:456-62. 
30. Tang Y, Luo J, Zhang W, Gu W. Tip60-dependent acetylation of p53 modulates the decision between cell-cycle arrest and apoptosis. Mol Cell. 2006;24:827-39.

31. Mrakovcic M, Kleinheinz J, Fröhlich LF. p53 at the Crossroads between Different Types of HDAC Inhibitor-Mediated Cancer Cell Death. Int J Mol Sci. 2019;20:2415.

32. Cao L, Zhao S, Yang Q, Shi Z, Liu J, Pan T, et al. Chidamide Combined With Doxorubicin Induced p53Driven Cell Cycle Arrest and Cell Apoptosis Reverse Multidrug Resistance of Breast Cancer. Front Oncol. 2021;11:614458.

33. Wagner T, Brand P, Heinzel T, Krämer OH. Histone deacetylase 2 controls $\mathrm{p} 53$ and is a critical factor in tumorigenesis. Biochim Biophys Acta. 2014;1846:524-38.

34. Kim EH, Koh D-I, Ryu YS, Park S-S, Hong S-W, Moon J-H, et al. Role of p53 in transcriptional repression of SVCT2. Mol Biol Rep. 2021;48:1651-8.

35. Roy S, Packman K, Jeffrey R, Tenniswood M. Histone deacetylase inhibitors differentially stabilize acetylated p53 and induce cell cycle arrest or apoptosis in prostate cancer cells. Cell Death Differ. 2005;12:482-91.

36. Azmi AS, Mohammad RM. Targeting cancer at the nuclear pore. J Clin Oncol. 2016;34:4180-2.

37. Azmi AS, Uddin MH, Mohammad RM. The nuclear export protein XPO1 - from biology to targeted therapy. Nat Rev Clin Oncol. 2021;18:152-69.

\section{Figures}
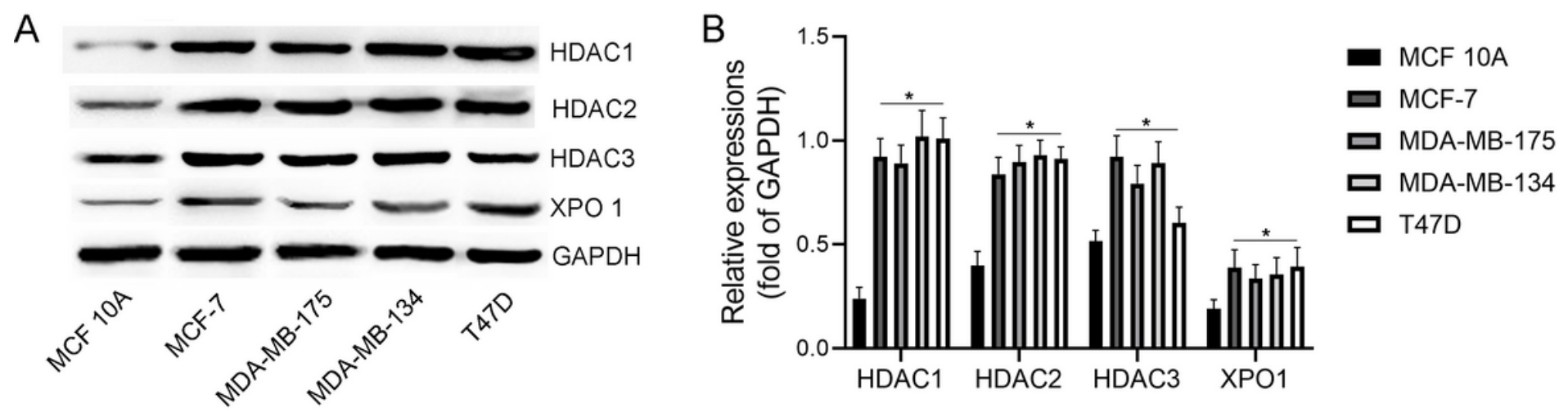

\section{Figure 1}

The protein expressions of HDAC1, HDAC2, HDAC3 and XP01 in cells of MCF 10A, MCF-7, MDA-MB-175, MDA-MB-134 and T47D. (A) The representative blots of HDAC1, HDAC2, HDAC3, XPO1 and GAPDH in cells of MCF 10A, MCF-7, MDA-MB-175, MDA-MB-134 and T47D respectively. (B) The histogram of semi quantitative analysis of HDAC1, HDAC2, HDAC3 and XPO 1 in cells in each cell lines (fold of GAPDH). The values were expressed as the means \pm S.E.M ( $n=6$ for each group). ${ }^{\star} \mathrm{p} \otimes 0.05$ vs. MCF $10 \mathrm{~A}$. 


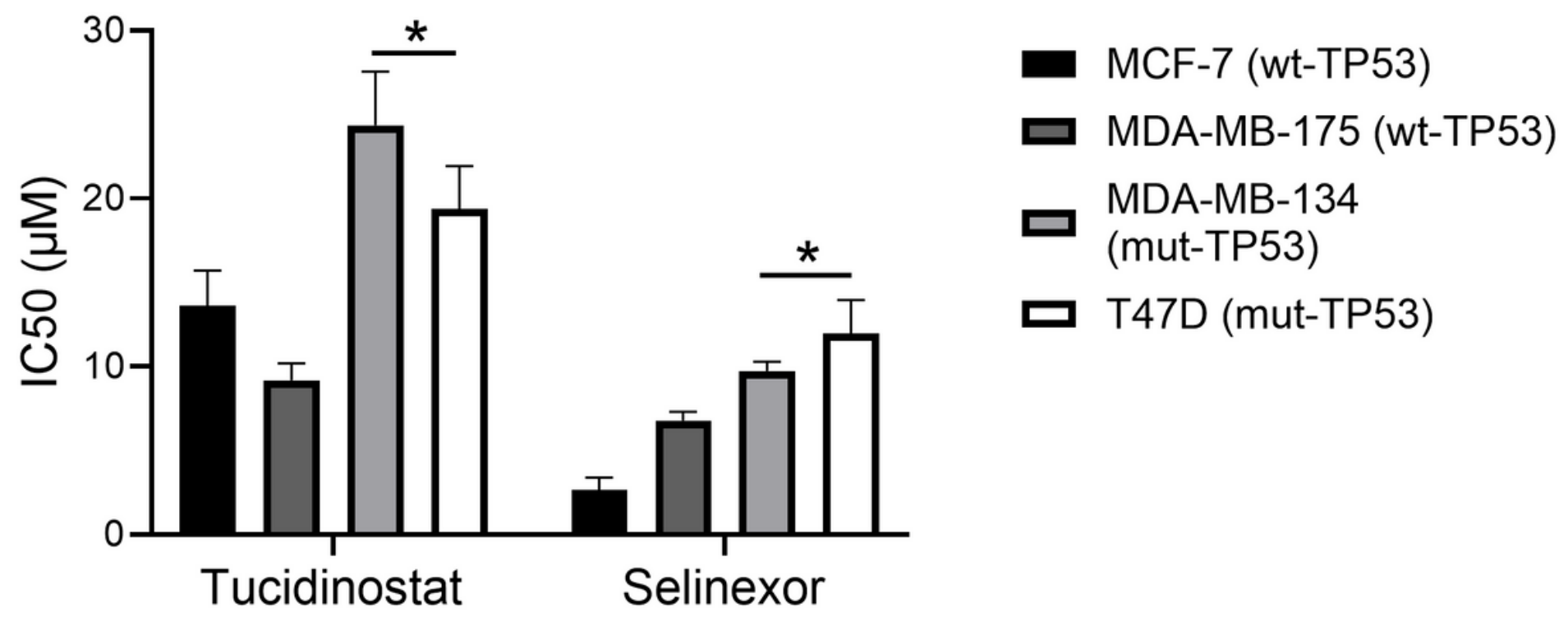

Figure 2

The comparison of IC50 values of tucidinostat and selinexor on MCF-7, MDA-MB-175, MDA-MB-134 and T47D. The cells were treated withtucidinostat or selinexor at different concentrations for $72 \mathrm{~h}$. Cell viability was measured using the MTT, and IC50values were then calculated using isobologram curves. The values were expressed as the means \pm S.E.M ( $n=6$ for each group). ${ }^{\star} p \otimes 0.05$ vs. MCF-7 or MDA-MB- 175 . 
A

MCF-7

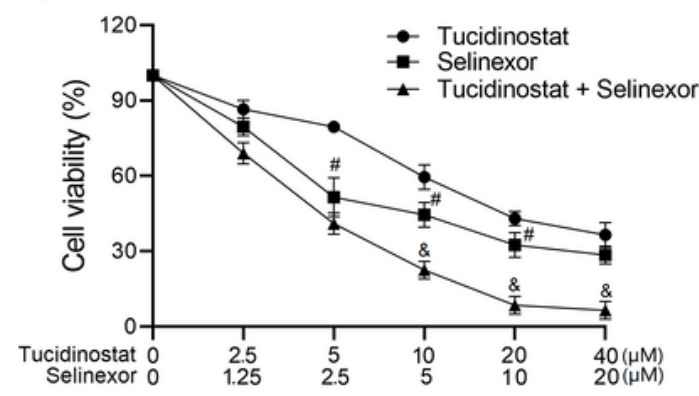

$\mathrm{Fa}-\mathrm{Cl}$ plot $\times$ Combinations

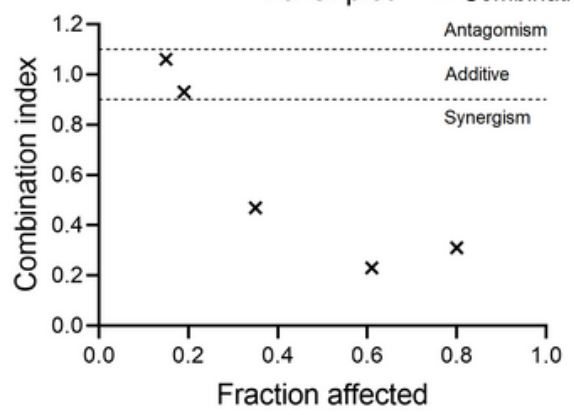

C

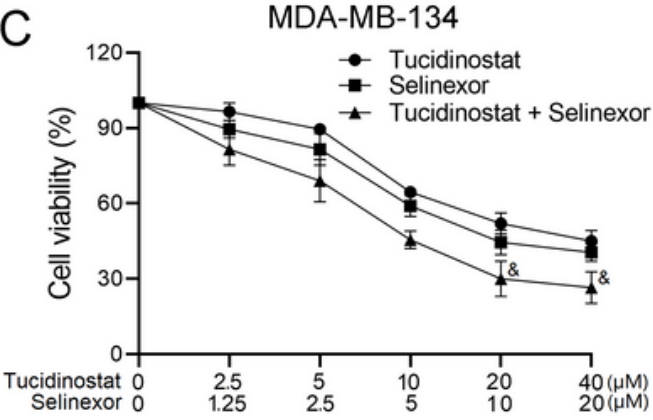

Fa-Cl plot $\times$ Combinations

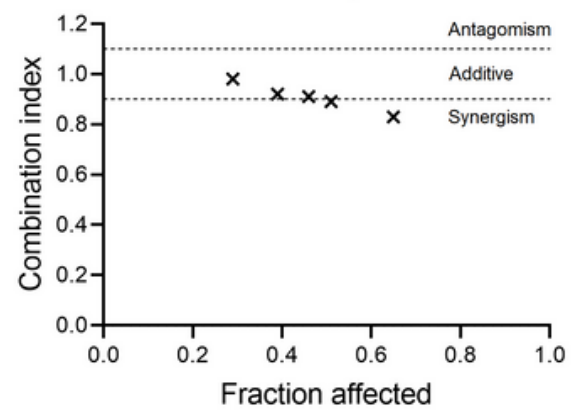

B
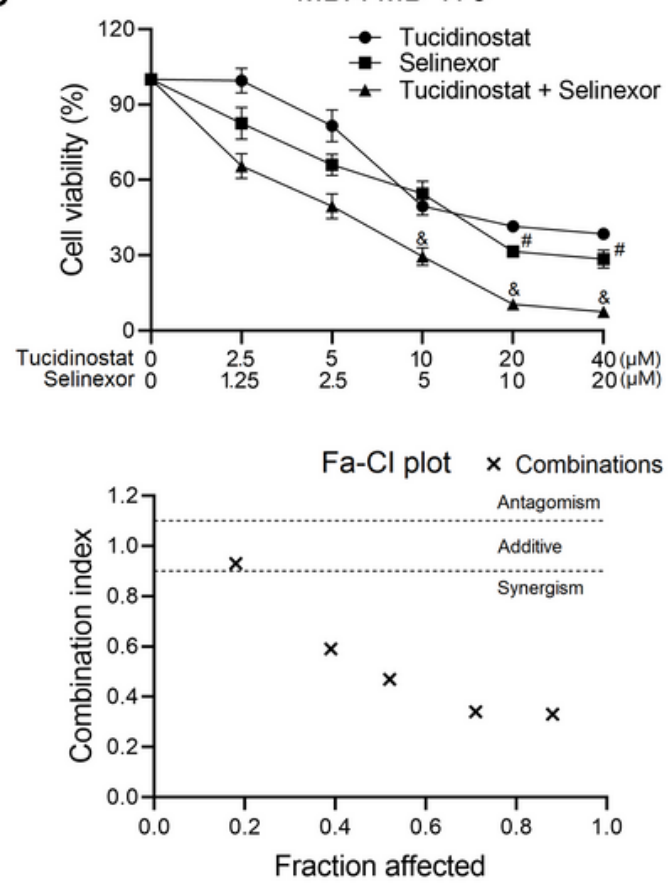

D

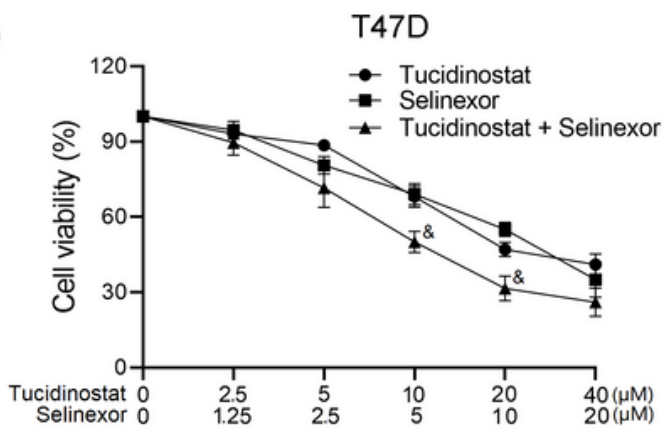

$\mathrm{Fa}-\mathrm{Cl}$ plot $\times$ Combinations

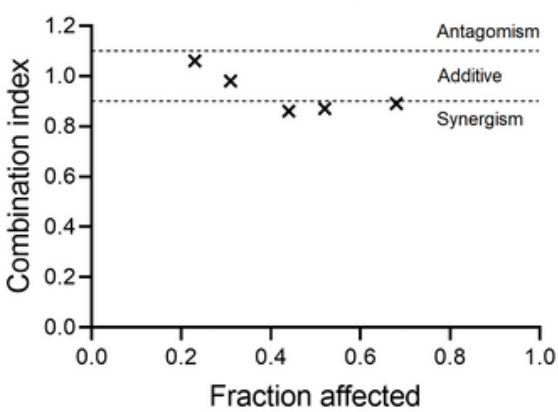

Figure 3

The effects of combination of tucidinostat and selinexor on cells viability of MCF-7, MDA-MB-175, MDAMB-134 and T47D. (A-D) Effects of different concentrations of tucidinostat, selinexor and their combination on cells viability of MCF-7,MDA-MB-175, MDA-MB-134 and T47D respectively after $72 \mathrm{~h}$ treatment. Combination indexes were calculated accordingly on the indicated concentrations of 
tucidinostat and selinexor. The values were expressed as the means \pm S.E.M ( $n=6$ for each group). \#p》

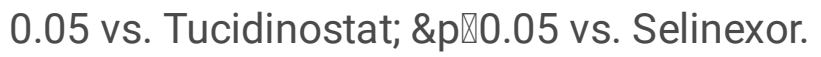
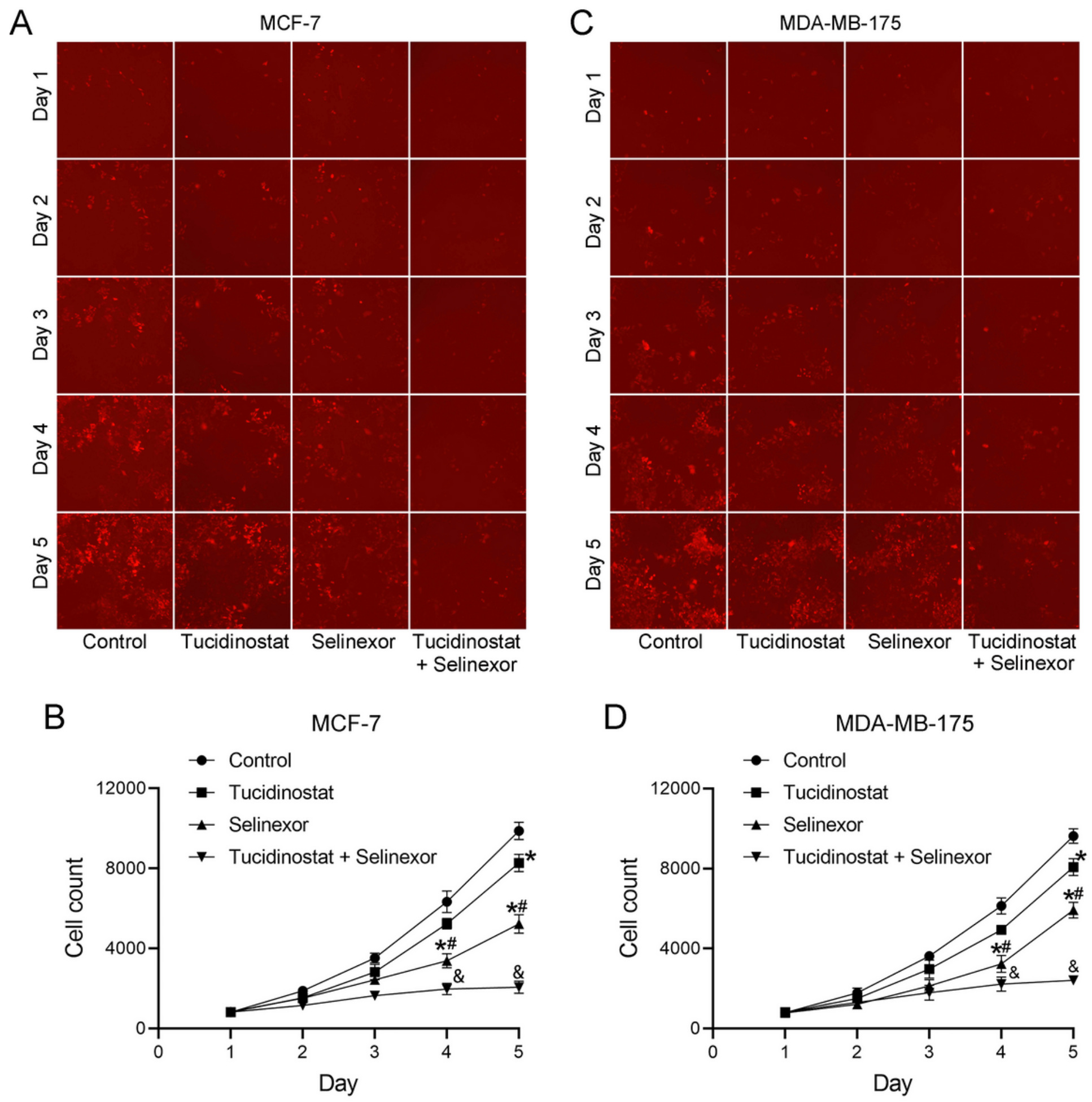

\section{Figure 4}

The effects of combination of tucidinostat and selinexor on the proliferation of wt-TP53 BC cells. The treatment concentrations of tucidinostat and selinexor here were $20 \mu \mathrm{M}$ and $10 \mu \mathrm{M}$, respectively. (A) The representative images of MCF-7 cells proliferation at different time points in each group (40x). (B) The 
cell counting curve of MCF-7. (C) The representative images of MDA-MB-175 cells proliferation at different time points in each group (40x). (D) The cell counting curve of MDA-MB-175. The values were expressed as the means \pm S.E.M ( $n=6$ for each group). ${ }^{\star} p<0.05$ vs. Control. \#p $\llbracket 0.05$ vs. Tucidinostat; \&p》 0.05 vs. Selinexor.
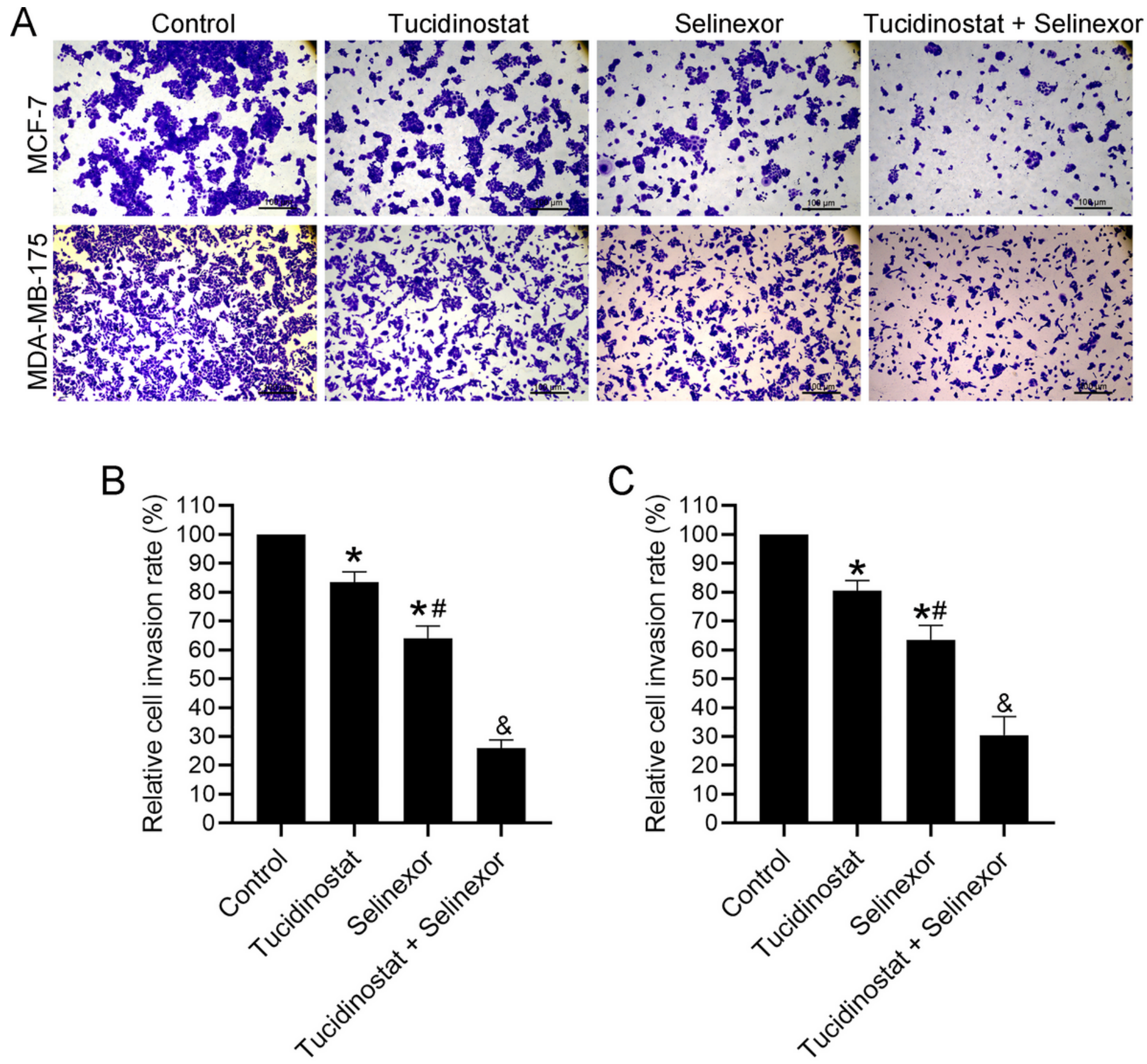

Figure 5

The effects of combination of tucidinostat and selinexor on the invasion of wt-TP53 BC cells. (A) The representative images of cells stained by crystal violet in the lower chamber after treatment with tucidinostat $(20 \mu \mathrm{M})$ and/or selinexor $(10 \mu \mathrm{M})$ for $24 \mathrm{~h}$. (B-C) The relative invasion rate of MCF-7 and MDA-MB-175. The values were expressed as the means \pm S.E.M ( $n=6$ for each group). ${ }^{*} p<0.05$ vs. Control. \#p $₫ 0.05$ vs. Tucidinostat; \&p $₫ 0.05$ vs. Selinexor. 

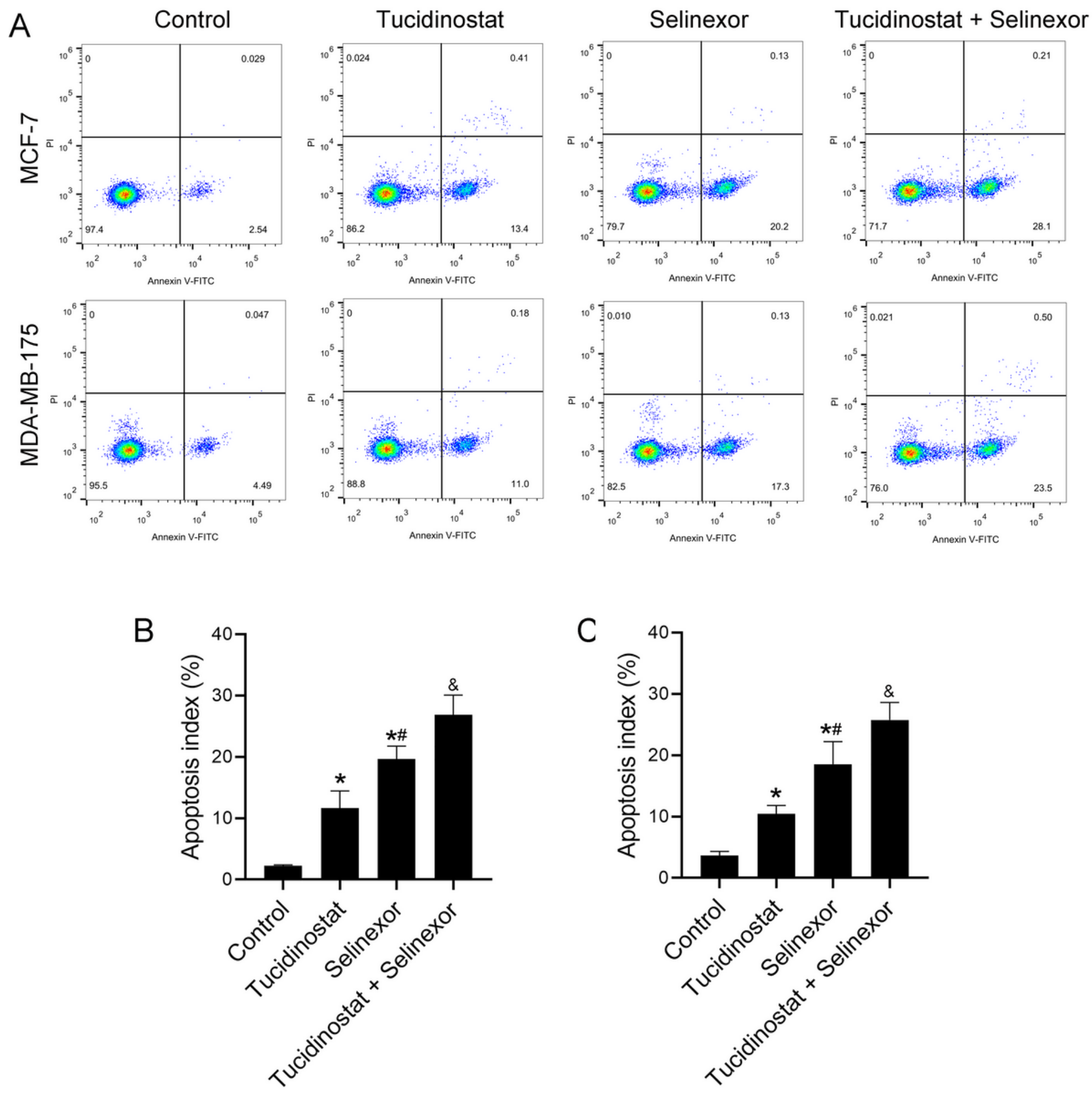

\section{Figure 6}

The effects of combination of tucidinostat and selinexor on apoptosis of wt-TP53 BC cells. (A) The representative images of flow cytometry in each experimental group of MCF-7 and MDA-MB-175 after treatment with tucidinostat $(20 \mu \mathrm{M})$ and/or selinexor $(10 \mu \mathrm{M})$ for $72 \mathrm{~h}$. (B) Apoptosis rates in each experimental group of MCF-7 cells. (C) Apoptosis rates in each experimental group of MDA-MB-175 cells. The values were expressed as the means \pm S.E.M ( $n=6$ for each group). ${ }^{*} p<0.05$ vs. Control. \#p $₫ 0.05$ vs. Tucidinostat; \&p\0.05 vs. Selinexor. 
A

MCF-7

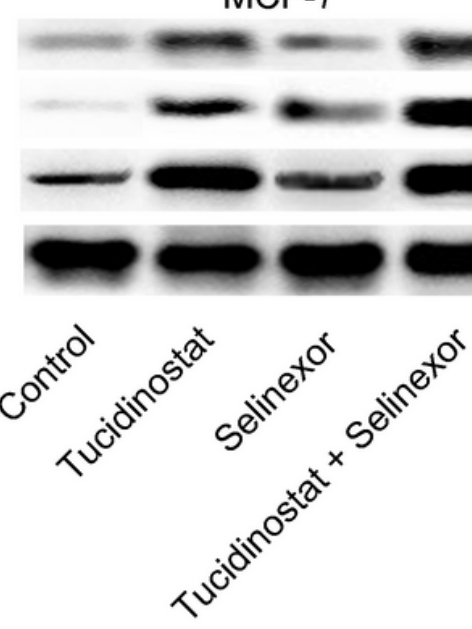

B

Acetyl-p53

Nuclear p53

Total p53

GAPDH
(3)
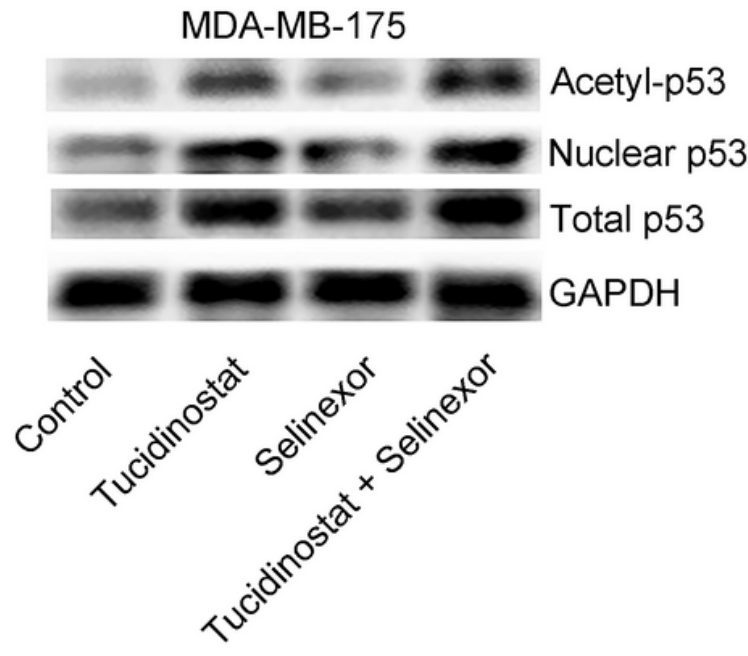
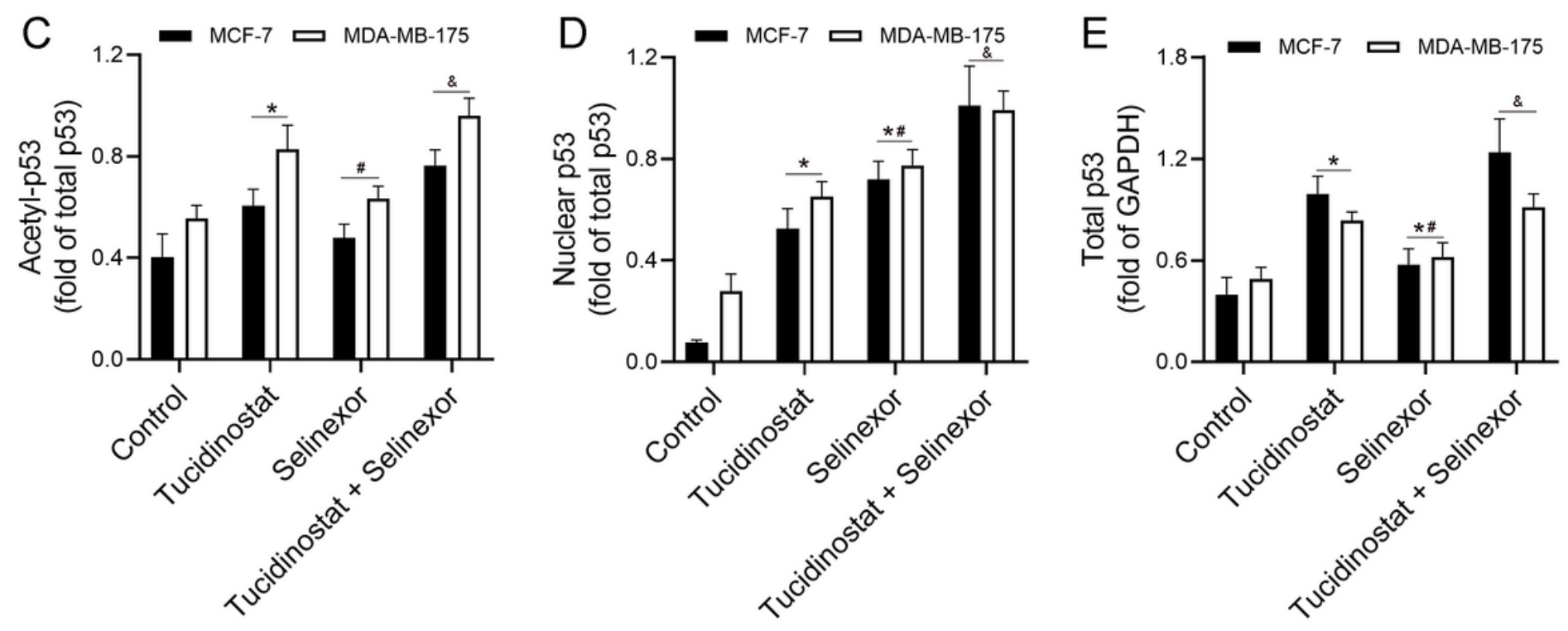

Figure 7

The effects of combination of tucidinostat and selinexor on expression of p53 in wt-TP53 BC cells. (A-B) The representative blots of acetyl-p53, nuclear p53, total p53 and GAPDH in cells of MCF-7 and MDA-MB175. (C-E) The histogram of semi quantitative analysis of acetyl-p53 (fold of total p53),nuclear p53 (fold of total p53) and total p53 (fold of GAPDH) in cells of MCF-7 and MDA-MB-175. The values were

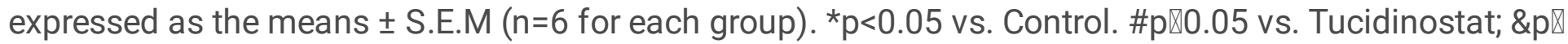
0.05 vs. Selinexor. 


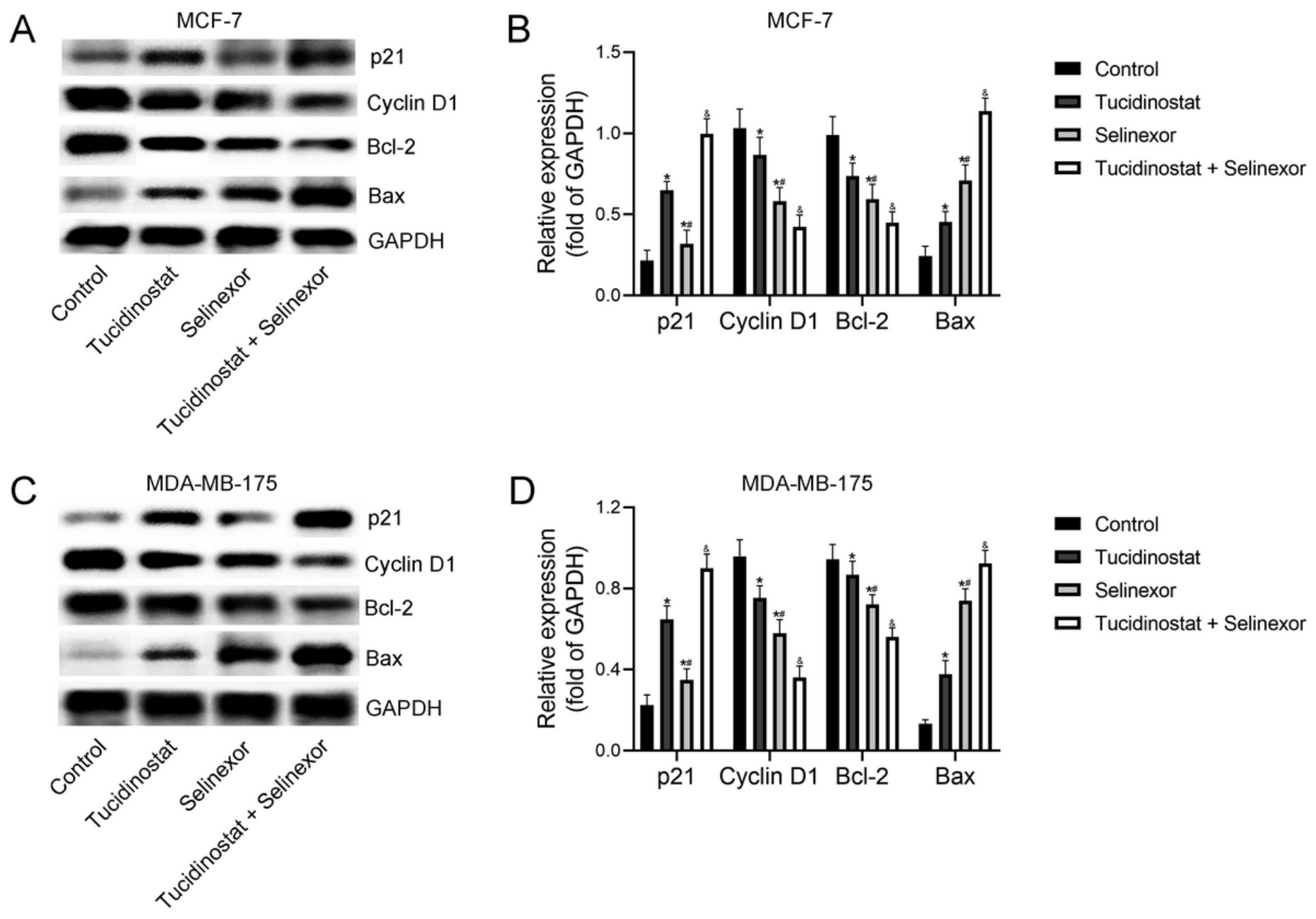

Figure 8

The effects of combination of tucidinostat and selinexor on protein expressions of $\mathrm{p} 21, \mathrm{Cyclin} \mathrm{D} 1, \mathrm{Bcl}-2$ and Bax in wt-TP53 BC cells. (A) The representative blots of p21, Cyclin D1, Bcl-2, Bax and GAPDH in cells of MCF-7. (B) The histogram of semi quantitative analysis of p21, Cyclin D1, Bcl-2 and Bax (fold of GAPDH) in MCF-7 cells. (C) The representative blots of p21, Cyclin D1, Bcl-2, Bax and GAPDH in cells of MDA-MB-175. (D) The histogram of semi quantitative analysis of p21, Cyclin D1, Bcl-2 and Bax (fold of GAPDH) in MDA-MB-175 cells. The values were expressed as the means \pm S.E.M ( $n=6$ for each group).

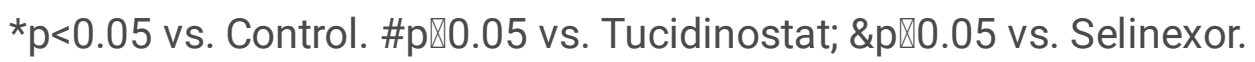

